\title{
Intestinal Permeability in Preterm Infants by Feeding Type: Mother's Milk Versus Formula
}

\author{
Sarah N. Taylor, Laura A. Basile, Myla Ebeling, and Carol L. Wagner
}

\begin{abstract}
Background and Objective: Intestinal permeability in preterm infants represents a critical balance between absorption of nutritional agents and protection from dangerous pathogens. This study identified the relationship between feeding type (human milk and formula) and intestinal permeability as measured by lactulose to mannitol ratio in preterm infants in the first postnatal month.

Study Design: Sixty-two preterm ( $\leq 32$ weeks of gestation) infants had assessment of feeding type and evaluation with enteral lactulose and mannitol administration and urinary measurement at three time points in the first postnatal month.

Results: Infants who received the majority of feeding as human milk $(>75 \%)$ demonstrated significantly lower intestinal permeability when compared to infants receiving minimal or no human milk ( $<25 \%$ or none) at postnatal days 7,14 , and 30 ( $p=0.02,0.02$, and 0.047 , respectively). When infants receiving any human milk were compared to infants receiving formula only, a significant difference existed at day 7 and day 14 but not for day $30(p=0.04,0.02$, and 0.15 , respectively). With evaluation over the complete study period, exclusively formulafed infants demonstrated a 2.8-fold higher composite median lactulose/mannitol ratio when compared with those who received any human milk. Infants who received $>75 \%$ of enteral feeding as mother's milk demonstrated a 3.8-fold lower composite median ratio when compared to infants receiving $<25 \%$ or no mother's milk. Conclusion: Preterm infant intestinal permeability was significantly decreased for those receiving human milk versus formula in a dose-related manner in the first postnatal month.
\end{abstract}

\section{Introduction}

$\mathbf{P}$ RETERM BIRTH REQUIRES infant development to continue in a foreign and often harmful extrauterine environment. The development of the gastrointestinal system plays a vital role in this time period as it serves both as a barrier to infectious materials and as a path for protective and nourishing substances. A precarious balance of intestinal permeability is required to promote infant growth and to avoid severe preterm infant disease such as necrotizing enterocolitis (NEC). Decreasing intestinal permeability is associated with gut maturation.

Lacutlose to mannitol $(\mathrm{L} / \mathrm{M})$ ratio measurement is a proven, effective index of intestinal permeability. ${ }^{1-4}$ Both lactulose and mannitol are absorbed as whole molecules by the human small intestine. Mannitol is small with a molecular radius of $0.4 \mathrm{~nm}$ and easily diffuses through the small intestine cell membrane. Lactulose with a radius of $0.52 \mathrm{~nm}$ is too large for intracellular diffusion and therefore relies on paracellular diffusion for intestinal absorption. ${ }^{5}$ The intestinal capacity for absorption of lactulose is a direct measure of the tightness of junctional complexes and thereby a measure of intestinal permeability. ${ }^{4-6}$ The absorption of mannitol is less dependent on intestinal integrity. Therefore, ingestion of lactulose and mannitol simultaneously controls for fluctuations in gastric emptying, intestinal fluid volume, and intestinal transit time, allowing direct measurement of the paracellular absorptive capacity of the gut. Attaining these molecules for measurement is easily performed with collection of urine. Both lactulose and mannitol have similar volumes of distribution within the body and similar renal clearance with excretion in the urine over a 5-hour period after enteral administration. ${ }^{1,2,5}$

Department of Pediatrics, Medical University of South Carolina, Charleston, South Carolina.

This study was presented in part at the 2006 Pediatric Academic Societies Meeting, San Francisco, California. 
Previous studies of preterm infant intestinal permeability have demonstrated permeability to be higher in preterm infants when compared to term infants but to not differ significantly by gestational age before 34 weeks of completed gestation. ${ }^{3,5,6}$ Intestinal permeability appears to decrease with initiation of enteral feeding. ${ }^{3,7}$ One study has shown increased permeability in preterm infants receiving formula feeds versus exclusive human milk feeds at 28 postnatal days, but not earlier or later. ${ }^{3}$ We identified no evaluation of the effect of human milk dose on preterm infant intestinal permeability in the literature. The aim for this study was to examine the effect of human milk volume on changes in intestinal permeability through the first postnatal month for preterm infants. We hypothesized that infants who received mother's milk would demonstrate more rapid decrease in $\mathrm{L} / \mathrm{M}$ ratio, as a marker of intestinal maturation, over time when compared to formula-fed infants.

\section{Subjects and Methods}

\section{Subjects}

Following approval from the Institutional Review Board for Human Subjects at the Medical University of South Carolina, Charleston, parents whose infants were admitted to the Neonatal Intensive Care Unit were approached for consent for their infant's participation if their infant met the following criteria: (1) $\leq 32$ weeks of gestation, (2) first feeding within 7 days after delivery, and (3) absence of major congenital anomalies including gastrointestinal malformation detected at birth. The study was conducted during a 3-year period.

\section{Study design}

This is a prospective study of preterm infants $\leq 32$ weeks of gestation who were followed for a 1-month study period as a part of an overall nutritional and intestinal maturity assessment.

\section{Study protocol}

Following informed consent, infants who met criteria were followed for a 1-month study period. Data were collected utilizing a standardized form developed by the investigators to ascertain information regarding prenatal history, delivery characteristics, health status, dose and volume of mother's milk received, initiation and duration of parenteral nutrition, use of human milk fortifier, and episodes of feeding intolerance that resulted in an infant being made NPO ("nothing by mouth") for at least a 24-hour period.

Initiation of feeds and advancement of feed volume were executed by a standard neonatal service protocol. The standard guidelines included consideration for initiation of feeds by postnatal day 1-4 depending on infant's condition and gestational age, advancement of feeds by $10-20 \mathrm{~mL} / \mathrm{kg} /$ day, and discontinuation of feeds for signs of feeding intolerance such as bilious residual, increased residual volume, emesis, abdominal distension, abdominal tenderness, or hematochezia or for clinical deterioration. Feeds were either maternal breastmilk and/or preterm infant formula started at $20 \mathrm{kcal} / \mathrm{oz}^{8,9}$ At $120-150 \mathrm{~mL} / \mathrm{kg} /$ day feeds, mother's milk was fortified at a 1:25 ratio, and formula was changed to preterm formula $(24 \mathrm{kcal} / \mathrm{oz}) .8,9$

\section{Intestinal permeability measurement}

Evaluation of intestinal permeability occurred on postnatal days 7-8, 14-15, and 28-30. Infants who had been without enteral feedings for $>72$ hours prior to a study day exited the study, with the intention to collect data on those premature infants who were without gastrointestinal compromise. If an infant was without enteral feedings for $<72$ hours on a study day, then the infant missed that sample collection, but continued participation in the study.

\section{Administration of lactulose/mannitol solution}

On each of the three study days, an infant was administered a $2 \mathrm{~mL} / \mathrm{kg}$ lactulose/mannitol solution (5 g of lactulose $+2 \mathrm{~g}$ of mannitol/100 $\mathrm{mL}$ ) via a nasogastric tube for two doses separated by three hours for a total dose of 100 $\mathrm{mg}$ of lactulose $/ \mathrm{kg}$ and $40 \mathrm{mg}$ of mannitol $/ \mathrm{kg}$. The lactulose/mannitol solution was administered by gavage with an enteral feed.

\section{Collection and processing of urine samples}

Starting at the time of the second dose of the lactulose/mannitol solution, urine was collected for a 6-hour period by urine bag. As urine loss does occur with collection by urine bag in preterm infants, the volume of urine loss was evaluated to assess any effect on the L/M ratio measurement. The urine samples were sent to the General Clinical Research Center at the Medical University of South Carolina where it was stored at $-80^{\circ} \mathrm{C}$ until analysis. In analysis, mannitol and lactulose were measured by enzymatic assay and gas chromatography by previously published techniques (Great Smokies Diagnostic Laboratory, Asheville, NC), 1,2 and a ratio of $\mathrm{L} / \mathrm{M}$ was calculated.

\section{Assignment of feeding type}

At each of the three study time points, dose and volume of mother's milk and formula over the interim period were recorded.

Feeding type was defined by two classification systems at each study point. One system defined an infant's feeding as receiving human milk feeds as majority $(>75 \%)$, partial $(25-75 \%)$, or minimal $(<25 \%) /$ none of enteral feeds. The other system identified an infant's feeding as receiving any human milk versus receiving formula only.

\section{Power and sample size calculations}

This study was part of an investigation of intestinal maturation and the role of human milk transforming growth factor- $\alpha$ in the process. The sample size was chosen to detect a clinically significant change of $30 \%$ in serum transforming growth factor- $\alpha$ concentrations between human milk-fed and formula-fed infants with power of $80 \%$ and detection of twosided type 1 significance at the 0.05 level.

\section{Statistical methods}

Statistical analyses were performed utilizing SAS software (SAS Institute, Cary, NC) and included the Shapiro-Wilkes test to assess for normal distribution. With non-normal distributions, comparisons were performed by Wilcoxon ranksum test using $t$ test approximation and two-sided test. Com- 
Table 1. Comparison of Median L/M Ratios (Range) Between Infants Receiving Human Milk as Majority (>75\%), Partial (25-75\%), or Minimal $(<25 \%) /$ None of Enteral Feeds

\begin{tabular}{lcccc}
\hline & \multicolumn{2}{c}{ Median L/M ratio (range) } \\
\cline { 2 - 5 } $\begin{array}{l}\text { Human milk in } \\
\text { enteral feeds }\end{array}$ & $\begin{array}{c}\text { Study time } 1 \\
(\mathrm{n}=47)\end{array}$ & $\begin{array}{c}\text { Study time } 2 \\
(\mathrm{n}=33)\end{array}$ & $\begin{array}{c}\text { Study time } 3 \\
(\mathrm{n}=20)\end{array}$ & $\begin{array}{c}\text { Composite } \\
\text { sum }\end{array}$ \\
\hline Majority & 0.067 & 0.169 & 0.132 & 0.298 \\
Partial & $(0.013-1.337)$ & $(0.011-0.585)$ & $(0.035-0.556)$ & 0.154 \\
Minimal/none & 0.1 & 0.09 & $(0.031-0.984)$ & $0.739-1.337)$ \\
& $(0.014-0.609)$ & $(0.025-2.178)$ & 0.315 \\
& 0.221 & 1.371 & $(0.247-1.887)$ & $(0.014-2.278)$ \\
\hline
\end{tabular}

Comparisons are by Wilcoxon rank-sum test. A significant difference $(p<0.05)$ was found at study times 1,2 , and 3 and the composite comparison for majority versus minimal/none. A significant difference $(p<0.05)$ was found at study time 3 and the composite comparison for partial versus minimal/none. No significant difference was demonstrated between majority and partial at any comparison. The study number $(n)$ represents the number of results available at each study time.

posite values were the sum of all values available for each subject. Evaluation for confounders was performed by dichotomizing variables with comparison by $\chi^{2}$ or Fisher's exact test. Numerical variables, such as birth weight, gestational age, days to full feeds, and formula volume, were dichotomized by median values. Significance was defined a priori as $p<0.05$ for all tests.

\section{Results}

Sixty-six infants were enrolled. Four of these infants were not fed prior to postnatal day 7 and were therefore excluded. The remaining 62 infants had a median (range) gestational age at birth of 29.2 weeks (24-32 weeks) and a median (range) birth weight of 1,225 g (601-2,103 g). The percentage of urine successfully collected did not affect the $\mathrm{L} / \mathrm{M}$ ratio result. The median L/M ratios and ranges for each comparison group at each study time point are given in Tables 1 and 2. Results are not available from every subject at all three time points because of lack of enteral feeding at the study time, transfer to another institution or discharge from the hospital, or inadequate urine volume obtained. Prior to study time point 3 (28-30 days), one infant developed NEC and exited the study. This subject had no L/M ratio results for study time points 1 and 2 secondary to lack of enteral feeding, for $<72$ hours, at those time points. At the first study visit, only one infant received $24 \mathrm{kcal} / \mathrm{oz}$ feeds. At the second study visit,
$51 \%$ of infants received fortified or $24 \mathrm{kcal} / \mathrm{oz}$ feeds, and this percentage rose to $88 \%$ at the third study visit.

At postnatal days 7 and 14, infants whose feedings were the majority human milk had a significantly lower median $\mathrm{L} / \mathrm{M}$ ratio when compared to infants receiving minimal or no human milk (day $7 p=0.02$, day $14 p=0.02$ ) (Table 1 ). However, no significant difference existed between median $\mathrm{L} / \mathrm{M}$ ratio when the subjects were compared as those who partially received mother's milk and those who received minimal or no mother's milk (Table 1). Also, at both postnatal day 7 and 14 a significant difference was found when comparing infants receiving any human milk with infants receiving formula (day $7 p=0.04$, day $14 p=0.02$ ) (Table 2 ).

At the third study time, human milk continued to demonstrate association with lower intestinal permeability, but the percentage of volume as human milk appeared to play a different role. When $\mathrm{L} / \mathrm{M}$ ratios were compared for infants receiving any human milk and infants receiving formula alone, no significant difference was seen (Table 2). However, when infants receiving the majority of feeds as human milk and a partial amount of feeds as human milk were compared to infants receiving minimal or no human milk, significantly lower $\mathrm{L} / \mathrm{M}$ ratios were found $(p=0.047$ and 0.049 , respectively) (Table 1).

With evaluation by composite sum over the 1-month time period, infants who received any human milk demonstrated significantly decreased $\mathrm{L} / \mathrm{M}$ ratios when compared to in-

Table 2. Comparison of Median L/M Ratios (Range) Between Infants Receiving Any Human Milk and Those Receiving Only Formula

\begin{tabular}{lcccc}
\hline & \multicolumn{4}{c}{ Median L/M ratio (range) } \\
\cline { 2 - 5 } Type of feeding & $\begin{array}{c}\text { Study time } 1 \\
(\mathrm{n}=47)\end{array}$ & $\begin{array}{c}\text { Study time } 2 \\
(\mathrm{n}=33)\end{array}$ & $\begin{array}{c}\text { Study time 3 } \\
(\mathrm{n}=20)\end{array}$ & Composite \\
\hline Any human milk & 0.076 & 0.167 & 0.178 & 0.343 \\
Formula only & $(0.013-1.337)$ & $(0.011-8.468)$ & $(0.031-1.791)$ & 0.347 \\
& 0.205 & 1.371 & $0.963-8.838)$ \\
& $(0.062-2.178)$ & $(0.218-30)$ & $(0.247-1.887)$ & $(0.576-32.525)$ \\
\hline
\end{tabular}

Comparisons are by Wilcoxon rank-sum test. A significant difference $(p<0.05)$ was found at study time 1 and study time 2 and in the composite comparison. The study number $(n)$ represents the number of results available at each study time. 
fants receiving formula only $(p=0.006)$ (Table 2$)$. This relationship also was seen when comparing infants who received the majority of feeds as human milk and infants who received partial human milk with infants who received minimal or no human milk ( $p=0.002$ and 0.3 , respectively) (Table 1). No significant difference in median $\mathrm{L} / \mathrm{M}$ ratios was found between infants receiving the majority of feeds as human milk and those receiving partial human milk feeds at any time point (Table 1).

In evaluation for potential confounders, $\mathrm{L} / \mathrm{M}$ ratios at each study time were divided into two groups defined as being either less than or equal to or greater than the median value for that time. The median ratio values were 0.1 for day 7 , 0.182 for day 14 , and 0.247 for days $28-30$. The two groups were then evaluated, at each study time, with no significant relationship found with birth weight, gestational age, or fortification of feeds (median, 14 days). L/M ratios were not significantly associated with achieving full feeds before or at 14 days (median day to full feeds) or after.

In evaluation of potential perinatal confounders, intestinal permeability at all three study times demonstrated no significant relationship with maternal steroids, maternal indomethacin therapy, or maternal antibiotics. In evaluation of potential postnatal confounders, L/M ratios had no significant association with antibiotic or indomethacin therapy. No infants received steroids prior to postnatal day 30 .

In evaluation of formula volume as a confounder, at postnatal day 7, a significant relationship was identified between formula volume above the study population median of 0.009 $\mathrm{L}$ (range, $0-1.865 \mathrm{~L}$ ) and the $\mathrm{L} / \mathrm{M}$ ratio $(p=0.04)$. Investigation for a similar relationship of intestinal permeability and formula volume below or above the median at postnatal day 14 (0.01 L; range, $0-1.998 \mathrm{~L}$ of formula) and at postnatal day 30 (0.462 L; range, $0-5.03 \mathrm{~L}$ of formula) demonstrated no significant association.

\section{Discussion}

In this cohort, feeding type was associated with gut permeability with a 2.8-fold higher composite median L/M ratio of formula-only fed infants compared with those who received human milk through the first postnatal month. Infants who received $>75 \%$ of enteral feeding as mother's milk demonstrated a 3.8-fold lower composite median L/M ratio than infants receiving $<25 \%$ or no mother's milk through the first postnatal month. Infants who received $25-75 \%$ of enteral feeds as mother's milk had a significantly lower composite median $\mathrm{L} / \mathrm{M}$ ratio than infants receiving $<25 \%$ or no mother's milk, but with a slightly less pronounced difference of 3.5-fold. In this population, human milk feeding was associated with decreased intestinal permeability from the end of the first postnatal week through the first month. The degree of difference in permeability appeared larger with higher human milk doses, but benefit was apparent with any human milk intake.

Receiving the majority of feedings as mother's milk was not significantly different from receiving partial feedings of mother's milk at any time, but only those receiving the majority of feedings as mother's milk had significantly decreased L/M ratios compared to infants receiving minimal or no human milk at postnatal days 7 and 14 . By postnatal day 30 , all infants receiving at least $25 \%$ of feeds as human milk had significantly lower intestinal permeability measurement than the group of infants receiving minimal or no human milk. In contrast, receiving any human milk was associated with significantly lower intestinal permeability measurements at postnatal day 7 and 14, but this comparison was no longer significant at postnatal day 30 . Therefore, human milk doses greater than $25 \%$ appear to play a role in the low intestinal permeability at the end of the first postnatal month.

Evaluation of the effect of formula intake revealed a significant association with formula volume and increased intestinal permeability at postnatal day 7 but not later in the first month. This finding suggests that a specific volume of formula may represent a toxic dose for the preterm intestine. Further evaluation of this relationship is warranted. Additionally, further evaluation of preterm infants with feeding difficulties is needed. In this study, L/M studies were not performed in infants with no enteral feeds at the study time. Evaluation of the human milk and formula relationship with intestinal permeability in infants with feeding tolerance or gastrointestinal compromise would add to these results.

In this study, the relationship of intestinal permeability and gestational age was evaluated. As shown in the previous studies of preterm infants less than 34 weeks of gestation, gestational age was not significantly associated with intestinal permeability. $3,5,6,10$ One study found similar intestinal permeability at birth but higher intestinal permeability at postnatal day 7 for the infants born before 28 weeks of gestation compared to infants born at or after 28 weeks of gestation. However, this study is complicated by the absence of enteral nutrition prior to postnatal day $8 .^{7}$

The previous studies of intestinal permeability in preterm infants include evaluations in the first postnatal week ${ }^{5,6}$ and over the first 24 days, ${ }^{7} 42$ days, ${ }^{10}$ and 50 days. ${ }^{3}$ In these five studies, evaluation of intestinal permeability was performed without regard to feeding status (feeding versus not feeding) and predominantly without regard to feeding type. Only one study compared infants receiving human milk versus formula. They found human milk feeds to be associated with decreased permeability at 28 days but not at 10 days or 50 days. $^{3}$

Two studies in term infants have evaluated the relationship of feeding type and intestinal permeability. ${ }^{4,11}$ Both studies demonstrated lower intestinal permeability in human milk-fed infants, but at dissimilar postnatal days. Lower intestinal permeability was seen with term breastfed infants compared to formula-fed infants at postnatal day 7 but not at postnatal day 1 or $30 .{ }^{4}$ A study of term infants over the first 6 postnatal days showed decrease in permeability to lactulose with initiation of human milk feedings but not formula feedings. ${ }^{11}$

Changes in preterm infant intestinal permeability based on type of feeding may play a critical role in the development of preterm infant disease. With past studies demonstrating fluctuations in intestinal permeability over the first week or first month, investigators have hypothesized that increased intestinal permeability is advantageous for the preterm infant. ${ }^{5,10}$ One study of 26-29 weeks gestational age infants demonstrated, by lactulose to rhamnose ratios, that intestinal permeability increased between 1-2 postnatal weeks and 3-4 postnatal weeks and then declined by 4-6 postnatal weeks. ${ }^{10}$ Another study of 26-30 weeks gestational 
age infants showed, by $\mathrm{L} / \mathrm{M}$ ratios, a mean increase in intestinal permeability from postnatal day 10 to day 28 with a decrease at day $50 .^{3}$ These results have lead to the theory that preterm infants display a period of increased intestinal permeability to promote absorption of larger immunological and growth-promoting molecules from human milk in an attempt to compensate for the shortened gestation. 5,10,12 Our results demonstrating an increase in the $\mathrm{L} / \mathrm{M}$ ratio for infants receiving formula with no appreciable fluctuation in the $\mathrm{L} / \mathrm{M}$ ratio for infants receiving predominantly human milk contradicts this theory.

Instead, our results support the theory that artificially fed infants lack human milk factors promoting intestinal tight junction closure and/or the artificial feedings cause mucosal damage leading to increased intestinal permeability. ${ }^{4,12,13}$ The difference in intestinal permeability between predominantly human milk-fed and predominately formula-fed infants peaked at 14 days and decreased by 30 days. With minimal variation in the intestinal permeability of predominantly human milk-fed infants, fluctuations in intestinal permeability for the predominately formula-fed infants accounted for the difference. The reason for the peak at 14 days is not easily identifiable but may relate to loss of protective mechanisms developed in utero prior to adaptation of the preterm intestine to postnatal exposures. The possibility that this association between increased intestinal permeability and formula-feeding may play a role in the increased incidence of NEC for formulafed infants is of great concern. ${ }^{14,15}$ NEC persistently threatens preterm infants with an incidence of approximately $7 \%$ in verylow-birth-weight $(<1,500 \mathrm{~g})$ infants. The pathophysiology of NEC is not entirely understood, but an immature intestinal mucosa and exposure to formula are factors in the development of this disease in preterm infants. Our finding that formula feeding and low human milk doses are associated with increased intestinal permeability provides further insight into the manifestation of this disease and the protection provided by human milk feeding.

\section{Conclusions}

As assessed by intestinal permeability changes in a cohort of healthy preterm infants $\leq 32$ weeks' gestation, human milk promotes intestinal closure earlier and more consistently than formula during the first postnatal month. The role of the portion of feeding provided as human milk appears to increase in importance through the month, with greater than minimal $(>25 \%)$ human milk required to see a significant relationship between human milk and intestinal permeability at postnatal day 30 .

\section{Acknowledgments}

We would like to thank Deanna Fanning for her careful review of the medical records to verify all clinical and laboratory data entered into the study database. We also thank all parents and their infants who participated in this study. This study was supported in part by grant MO1 RR001070 from the National Center for Research Resources, National Institutes of Health.

\section{Disclosure Statement}

No competing financial interests exist.

\section{References}

1. Behrens RH, Docherty $\mathrm{H}$, Elia M, et al. A simple enzymatic method for the assay of urinary lactulose. Clin Chim Acta 1984;137:361-367.

2. Lunn PG, Northrop CA, Northrop AJ. Automated enzymatic assays for the determination of intestinal permeability probes in urine. 2. Mannitol. Clin Chim Acta 1989;183: 163-170.

3. Shulman RJ, Schanler RJ, Lau C, et al. Early feeding, antenatal glucocorticoids, and human milk decrease intestinal permeability in preterm infants. Pediatr Res 1998;44: 519-523.

4. Catassi C, Bonucci A, Coppa V, et al. Intestinal permeability changes during the first month: effect of natural versus artificial feeding. I Pediatr Gastroenterol Nutr 1995;21:383-386.

5. Weaver LT, Laker MF, Nelson R. Intestinal permeability in the newborn. Arch Dis Child 1984;59:236-241.

6. Van Elburg RM, Fetter WP, Bunkers CM, et al. Intestinal permeability in relation to birth weight and gestational and postnatal age. Arch Dis Child Fetal Neonatal Ed 2003;88: F52-F55.

7. Rouwet EV, Heineman E, Buurman WA, et al. Intestinal permeability and carrier-mediated monosaccharide absorption in preterm neonates during the early postnatal period. Pediatr Res 2002;51:64-70.

8. Mead Johnson Nutritionals Product Handbook. Mead Johnson and Company, Evansville, IN, 2006.

9. Ross Products Handbook. Abbott Laboratories. Abbott Park, IL, 2006.

10. Beach R, Menzies IS, Clayden GS, et al. Gastrointestinal permeability changes in the preterm neonate. Arch Dis Child 1982;57:141-145.

11. Weaver LT, Laker MF, Nelson R, et al. Milk feeding and changes in intestinal permeability and morphology in the newborn. J Pediatr Gastroenterol Nutr 1987;6:351-358.

12. Insoft RM, Sanderson IR, Walker WA. Development of immune function in the intestine and its role in neonatal diseases. Pediatr Clin North Am 1996;43:551-571.

13. Veereman-Wauters G. Neonatal gut development and postnatal adaptation. Eur J Pediatr 1996;155:627-632.

14. Weaver LT, Laker MF, Nelson R. Enhanced intestinal permeability in preterm babies with bloody stools. Arch Dis Child 1984;59:280-281.

15. Caplan MS, Hedlund E, Adler L, et al. Role of asphyxia and feeding in a neonatal rat model of necrotizing enterocolitis. Pediatr Pathol 1994;14:1017-1028.

Address reprint requests to: Sarah N. Taylor, M.D. Department of Pediatrics Medical University of South Carolina 165 Ashley Avenue Charleston, SC 29425

E-mail: taylorse@musc.edu 